\title{
Evaluating the living donor: expansion by innovation
}

\author{
Chung-Mau Lo'
}

Received: 1 December 2015 / Accepted: 5 February 2016/Published online: 26 February 2016

(C) Asian Pacific Association for the Study of the Liver 2016

Living donor liver transplantation (LDLT) provides an alternative for patients with end-stage liver disease to receive a life-saving transplant operation. As compared to deceased donor liver transplantation, LDLT is both surgically and ethically more challenging, as the operation involves taking a partial liver graft from a healthy living donor. The evaluation and selection of a living donor is a more complicated process which can be no less controversial than the donor operation itself. There are three main objectives. The first is to determine that a part of the donor's liver can be used reliably as a graft for the recipient. The second is to ensure the safety of the donor. The final objective is to assess the outcome of the recipient including the risks and benefits as compared to a deceased donor liver transplant. To ensure the safety of the operation, particularly for the donor, the pretransplant evaluation of a potential living donor according to a planned protocol and criteria is mandatory. There is extremely wide variation in the practice of donor evaluation among different transplant centers because of differences in the availability of deceased donor grafts, experience in LDLT, prevalence of various diseases, and socio-cultural factors.

Metabolic syndrome, which includes a combination of obesity, diabetes, hypertension and dyslipidemia, represents a common risk factor for premature cardiovascular disease [1]. It is also closely but not causally related to nonalcoholic fatty liver disease (NAFLD) [2] which spans from steatosis to steatohepatitis. Hence, the presence of

Chung-Mau Lo

chungmlo@hku.hk

1 Department of Surgery, The University of Hong Kong, Queen Mary Hospital, 102 Pokfulam Road, Hong Kong, China metabolic syndrome may potentially increase the operative risk of a living liver donor for both reasons. In this issue of the journal, Al-hamoudi et al. summarizes the outcome of 1065 potential donors who were evaluated for living liver donation at the King Faisal Hospital in Saudi Arabia [3], and identified elements of metabolic syndrome as the most common reasons for rejecting potential living liver donors. In potential donors for adults, the donation rate was only $13 \%$, with the most common reasons for rejection being a body mass index $>28$ in $28 \%$ and diabetes in $19.2 \%$. Hepatic steatosis $>10 \%$ on radiologic imaging or liver biopsy was the reason for rejection in an additional $8 \%$ of the donors. As a high volume center with an average of 40 LDLTs per year, which accounted for over $50 \%$ of the annual case load of liver transplants, this experienced group from King Faisal Hospital has adopted a very cautious approach in donor selection with an upper age limit set at 45 years, a body mass index (BMI) under 28 , and a policy of routine liver biopsy with the aim of accepting only donors with steatosis $<10 \%$. The reasons as well as the consequences of such a cautious approach are obvious when considering the prevalence of metabolic syndrome in Saudi Arabia which is 10-15\% higher than in most developed countries [4].

It is interesting to compare this experience from Saudi Arabia with the Hong Kong experience in a predominantly Chinese community [5] and the American experience from the US adult-to-adult living donor liver transplantation (A2ALL) study [6], which reported much higher donation rates, of 32 and $40 \%$, respectively. When making such a comparison, a basic revelation is the fact that many selection criteria are arbitrarily set solely on the basis of donor safety and without any scientific basis. For example, the upper age limit for living liver donors is 45 in Saudi Arabia [3] but 60 in both Hong Kong and the US [5, 6]. 
The average donor age is under 28 years in Saudi Arabia as compared to 35-37 years in the Chinese and American studies. Interestingly, such a strict age limit in Saudi Arabia would have excluded older donors who may have a higher incidence of comorbidities, metabolic syndrome and NAFLD. As a result, extending the age limit from 45 to 60 in Saudi Arabia may potentially increase the total number of donors but paradoxically further decrease the overall rate of donation.

Similar to age, the effect of obesity per se on the safety of donor hepatectomy is debatable, and the upper BMI limit for living liver donation is arbitrary. As a country in which obesity is not uncommon, Saudi Arabia declines donors with a BMI $>28$ [3], and this has led to the rejection of over one-quarter of all potential liver donors. In contrast, in Hong Kong and the US [5, 6], a high body mass index by itself is not regarded as a contraindication for living donations but instead calls for the need for more tests for underlying comorbidities and associated NAFLD. In the US experience, for example, $15 \%$ of the donors accepted for donation had a BMI of 30 or higher. Since a high body mass index is predictive of hepatic steatosis [7], a liver biopsy, if not routinely done, is necessary in an obese donor. Variable degrees of up to $20 \%$ steatosis may be acceptable for right liver donation [7], but the presence of non-alcoholic steatohepatitis (NASH) should be regarded as an absolute contraindication [8]. NASH is characterized histologically not only by steatosis but also by hepatocellular injury and fibrosis, and it is obvious that resection of nearly two-thirds of such an unhealthy liver would put the donor at high risk. Indeed, there has been a donor death attributed to NASH in Japan [8].

The growing epidemic of metabolic syndrome worldwide may become a greater obstacle in living donation not only in Saudi Arabia but also in many other parts of the world. Governmental effort to control this epidemic is unlikely to have any impact on the donor pool in the near future. Yet, the cautious approach of the Saudi group regarding metabolic syndrome in the selection of liver donors is justified, since donor safety is the primary concern in LDLT. There are several other barriers that have already been overcome to expand the donor pool without increasing donor risks. First, experiences from Hong Kong where HBV is endemic, and where more than $50 \%$ of living donors are seropositive for antibodies to hepatitis core antigen (anti-HBc), have already indicated the safety of right liver donation in these donors if their serum is negative for hepatitis B surface antigen [9]. Despite the fact that anti-HBc positive donors are older, there is no difference in the operative outcome and no donors had reactivation of hepatitis or other liver problems on long-term follow up. Second, the ABO-blood group barrier has already been overcome either by the rituximab-based special immune suppression protocol [10] or using a strategy of donor exchange [11]. Third, with technical advances and better experience, donors with biliary and vascular anatomic variations can now be accepted for donation $[12,13]$. Such anatomic variants in donors may slightly increase the risks of the recipient but not the donor and are no longer considered as contraindications for LDLT.

In the Saudi Arabia experience, insufficient volume either of the liver remnant for the donor or of the graft in the recipient was the reason for rejecting as many as $17 \%$ of the donors. This is very high when compared to our experience in Hong Kong, especially when females (hence with smaller liver size) constitute nearly $60 \%$ of the donors in Hong Kong and less than $20 \%$ of those in Saudi Arabia. The adequacy of the graft size is the ultimate challenge in adult LDLT which involves a trade-off between donor and recipient safety. While a small liver graft may put the recipient at risk for small-for-size syndrome, taking a larger graft may leave the donor with a small liver remnant with associated safety issues. Compared to a left liver donation, a right liver donation provides the recipient with a graft twice as large but puts the donor at 5 times the risk of mortality [14]. The minimum graft size requirement for successful LDLT has been set at $40 \%$ for graft-weight-to-recipient-standard-liver-volume (GW/SLV) ratio [15] or $1 \%$ for graft-to-recipient-weight ratio (GRWR) [16]. With more experience and a better understanding of the mechanism of graft injury in smallfor-size syndrome, there is evidence that this minimum graft size safety limit can be lowered and a higher proportion of LDLT using left liver graft could be performed [17]. We have previously estimated that, for every $5 \%$ reduction in the minimum requirement for GW/SLV ratio, the proportion of adult LDLT using left liver graft would be doubled, while if the minimum GW/SLV ratio for successful LDLT could be reduced to $25 \%$, a left liver graft might be used in two-thirds of all adult LDLTs [18]. Successfully extending the selection criteria in this direction will not only expand the donor pool by removing inadequate size of the graft or liver remnant as a reason for rejecting a donor but, more importantly, it will also improve the safety of the donor operation which is the key element to consider as we try to expand the donor pool and develop LDLT.

\section{References}

1. Isomaa B. A major health hazard: the metabolic syndrome. Life Sci. 2003;73:2395-2411.

2. Loria P, Lonardo A, Carulli L, Verrone AM, Ricchi M, Lombardini S, et al. Review article: the metabolic syndrome and non- 
alcoholic fatty liver disease. Aliment Pharmacol Ther. 2005;22(Suppl. 2):31-36.

3. Al-hamoudi W, Abaalkhail F, Bendahmash A, Allam N, Hegab B, Elsheikh Y, et al. The impact of metabolic syndrome and prevalent liver disease on living donor liver transplantation: a pressing need to expand the pool. Hepatol Int. (in press).

4. Mabry RM, Reevest MM, Eakin EG, Owen N. Gender differences in prevalence of the metabolic syndrome in Gulf Cooperation Council Countries: a systematic review. Diabet Med. 2010;27(5):593-597.

5. Chan SC, Fan ST, Liu CL, Lo CM, Lam BK, Lee EW. Working up donors for high-urgency and elective adult-to-adult live donor liver transplantation. Liver Transpl. 2007;13(4):509-515.

6. Trotter JF, Wisniewski KA, Terrault NA, Everhart JE, Kinkhabwala M, Weinrieb RM, Fair JH, Fisher RA, Koffron AJ, Saab S, Merion RM, the A2ALL Study Group. Outcomes of donor evaluation in adult-to-adult living donor liver transplantation. Hepatology. 2007;46(5):1476-1484.

7. Siriwardana RC, Chan SC, Chok KSH, Lo CM, Fan ST. Effects of donor steatosis on liver biochemistry and significance of body mass index in predicting steatosis. HPB. 2012;14(9):619-624.

8. Yamamoto K, Takada Y, Fujimoto Y, Haga H, Oike F, Kobayashi N, et al. Nonalcoholic steatohepatitis in donors for living donor liver transplantation. Transplantation. 2007;83(3): 257-262.

9. Lo CM, Fan ST, Liu CL, Yong BH, Wong Y, Ng IO, et al. Safety and outcome of hepatitis B core antibody-positive donors in right-lobe living donor liver transplantation. Liver Transpl. 2003;9(8):827-832.

10. Ikegami T, Taketomi A, Soejima Y, Yoshizumi T, Uchiyama H, Harada N, et al. Rituximab, IVIG, and plasma exchange without graft local infusion treatment: a new protocol in $\mathrm{ABO}$ incompatible living donor liver transplantation. Transplantation. 2009;88(3):303-307.

11. Chan SC, Lo CM, Yong BH, Tsui WJ, Ng KK, Fan ST. Paired donor interchange to avoid $\mathrm{ABO}$-incompatible living donor liver transplantation. Liver Transpl. 2010;16(4):478-481.

12. Guler N, Dayangac M, Yaprak O, Akyildiz M, Gunay Y, Taskesen F, et al. Anatomical variations of donor portal vein in right lobe living donor liver transplantation: the safe use of variant portal veins. Transpl Int. 2013;26(12):1191-1197.

13. Song GW, Lee SG, Hwang S, Sung GB, Park KM, Kim KH, et al. Preoperative evaluation of biliary anatomy of donor in living donor liver transplantation by conventional nonenhanced magnetic resonance cholangiography. Transpl. Int. 2007;20(2):167-173.

14. Barr ML, Belghiti J, Villamil FG, Pomfret EA, Sutherland DS, Gruessner RW, et al. A report of the Vancouver Forum on the care of the live organ donor: lung, liver, pancreas, and intestine data and medical guideline. Transplantation. 2006;81(10):1373-1385.

15. Lo CM, Fan ST, Liu CL, Chan JK, Lam BK, Lau GK, et al. Minimum graft size for successful living donor liver transplantation. Transplantation. 1999;68(8):1112-1116.

16. Kiuchi $T$, Kasahara $M$, Uryuhara $K$, Inomata $Y$, Uemoto $S$, Asonuma $\mathrm{K}$, et al. Impact of graft size mismatching on graft prognosis in liver transplantation from living donors. Transplantation. 1999;67(2):321-327.

17. Ogura Y, Hori T, El Moghazy WM, Yoshizawa A, Oike F, Mori A, et al. Portal pressure $<15 \mathrm{~mm} \mathrm{Hg}$ is a key for successful adult living donor liver transplantation utilizing smaller grafts than before. Liver Transpl. 2010;16(6):718-728.

18. Chan SC, Fan ST, Chok KS, Sharr WW, Dai WC, Fung JY, et al. Increasing the recipient benefit/donor risk ratio by lowering the graft size requirement for living donor liver transplantation. Liver Transpl. 2012;18(9):1078-1082. 